\title{
Potentiating cancer immunotherapies
}

\author{
The repertoire of new preclinical strategies for fighting tumours that resist or evade immunotherapy drugs and \\ cellular immunotherapies is widening.
}

M alignant tumours can resist the mechanisms of action of immunotherapy drugs and the effects of adoptive cell therapies, or may evolve to circumvent them. The strategies of resistance and evasion are, unfortunately, varied and plenty. First, specific signalling pathways can be activated, silenced or rewired to induce, via the lower expression of chemokines attracting immune cells, the exclusion of $\mathrm{T}$ cells from the tumour microenvironment (for example, enhanced signalling by the kinase PI3K via the loss of the gene encoding PTEN is associated with resistance to immune-checkpoint therapies). Second, tumour cells can constitutively express specific cell-surface ligands (such as the programmed-cell-death-receptor ligand $1(\mathrm{PD}-\mathrm{L} 1))$ that inhibit cytotoxic $\mathrm{T}$ cells. Third, tumours can lack antigenic mutations, downregulate antigen expression, or epigenetically alter the expression of genes associated with the processing and presentation of tumour antigens (cancer cells can downregulate the cell-surface expression of major histocompatibility complexes, which are required to present tumour antigens to T cells). Fourth, tumour cells can secrete ligands for attracting cells that suppress T-cell responses (such as myeloid-derived suppressor cells, regulatory T cells and tumour-associated type-II macrophages).

Some of these resistance or escape mechanisms are not fully understood, and, surely, more will be discovered. Moreover, tumours can also acquire resistance to immunotherapies via mechanisms extrinsic to the tumour cells. For instance, chronic stimulation of $\mathrm{T}$ cells by tumour antigens leads to T-cell exhaustion, which results in the sustained upregulation of T-cell checkpoint inhibitory receptors (such as PD-L1 and the T-cell immunoreceptor with immunoglobulin and ITIM domain (TIGIT)). And antitumour immune responses can, in turn, switch on genes and pathways that inhibit them; for example, effector T cells can increase the production of interferons, which (via enzymes such as indoleamine 2,3-dioxygenases (IDO)) can promote the secretion of metabolites (such as kynurenine, a metabolite of the amino acid tryptophan) that inhibit effector-T-cell function. Furthermore, the microenvironment of solid tumours

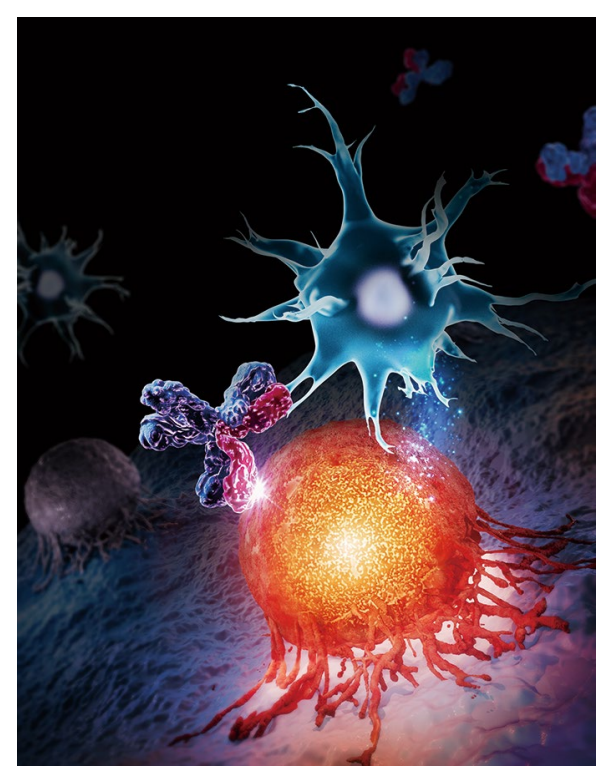

Credit: Longchao Liu and Yang-Xin Fu, University of Texas Southwestern Medical Center

hampers the infiltration of T cells via hypoxic zones, abnormal vasculature, stromal walls and other physical barriers.

The presence of tumours that adapt or acquire resistance to checkpoint inhibitors and other immunotherapy drugs, and to adoptive cell therapies such as T cells with chimeric antigen receptors (CARs), is associated with mixed clinical responses and with tumour relapse and cancer progression following an initial response. Achieving long-term survival in a greater fraction of patients and for a broader range of cancers requires that immunotherapy stimulates responses that are adequately potent, safe and durable (Nat. Biomed. Eng. 5, 377-378 (2021)).

One rather obvious strategy for enhancing the outcomes of immunotherapies is to use combinations of them to decrease the overall chance that tumours resist or evade multiple mechanisms of action. In particular, combinations of checkpoint inhibitors (such as antibodies for PD-L1 and TIGIT) and checkpoint inhibitors combined with antibodies for co-stimulatory targets (such as OX40), metabolic modulators (such as IDO inhibitors) or oncolytic viruses, or with adoptive cell therapies, cancer vaccines, targeted therapies, chemotherapies or radiation therapies, are all being tested in patients, and a few have been approved by regulators. However, combination immunotherapies are generally more complex to develop, manufacture, administer and assess (the optimal dosing and timing and sequence of administration need to be determined) and come with heightened risks of toxicity and off-target effects.

Monotherapies and combination therapies that implement strategies to reduce off-tumour toxicity and to counter the resistance and evasion mechanisms of tumours could lead to more durable and potent antitumour responses. This issue of Nature Biomedical Engineering includes a few Articles that are indicative of the widening repertoire of strategies for combating adaptive or acquired resistance to immunotherapies.

Because many antigens targeted by immunotherapies are not exclusively expressed by tumour cells, on-target off-tumour toxicity is one of the main challenges of adoptive cell therapies. Yingxiao Wang, Shu Chien and colleagues report in an Article that the activation of cytotoxic T cells genetically engineered with a CAR cassette under the control of a heat-shock protein promoter can be effectively confined within tumours using heat generated by short pulses of focused ultrasound under the guidance of magnetic resonance imaging. Similarly, Gabriel Kwong and colleagues show that the activity of CAR T cells engineered to express a stimulatory cytokine or a bispecific T-cell engager (to target antigen-negative tumour cells and thus mitigate tumour evasion by antigen escape) under the control of a thermal gene switch (also involving heat-shock elements) can be constricted within tumours by elevating their temperature by a few degrees using intravenously injected plasmonic nanorods and a near-infrared laser under the guidance of a thermal camera.

Bispecific T-cell engagers can also be designed to target dendritic cells (rather than tumour cells) to rejuvenate cytotoxic $\mathrm{T}$ cells, as Yang-Xin Fu, Jian Qiao and colleagues report in an Article also in this issue. Specifically, they show that bispecific antibodies (pictured) binding PD-L1 on dendritic cells and the T-cell receptor CD3 (which conventional 
bispecific T-cell engagers also target) led to durable T-cell cytotoxicity.

However, some tumours, such as pancreatic ductal adenocarcinomas, are lowly immunogenic and generate an immunosuppressive microenvironment. To enhance the infiltration of adoptive $\mathrm{T}$ cells into pancreatic tumours, Sebastian Kobold and colleagues engineered antigen-specific cytotoxic T cells to overexpress a $\mathrm{C}-\mathrm{X}-\mathrm{C}$ chemokine receptor whose ligand is highly expressed by pancreatic cancer cells. As noted by Leonid Cherkassky and Prasad S. Adusumilli in an accompanying News and Views article, the therapy is expected to be relatively safe owing to the low expression levels of the ligand by healthy pancreatic tissue.

Cancer stem cells can confer adaptive resistance. Natural killer (NK) cells can, in principle, kill these quiescent cells. Yet NK cells do not naturally recognize specific antigens, and producing NK cells with specific CARs is laborious and complex. Yee Shok Cho and co-authors report in this issue that, by using pluripotency transcription factors and an optimized reprogramming medium, NK cells (with or without an antigen-specific CAR and with the rare yet therapeutically more potent CD56 $6^{\text {bright }}$ CD16 ${ }^{\text {bright }}$ phenotype) can be directly obtained from human somatic cells.

Two more Articles in this issue report strategies for overcoming acquired resistance to checkpoint inhibitors. Yong $\mathrm{Lu}$ and colleagues show that tumour cells and immunosuppressive cells can be depleted concurrently via a monoclonal antibody binding a cell-surface enzyme (CD73) upregulated on tumour cells as well as on regulatory $\mathrm{T}$ cells, myeloid-derived suppressor cells and tumour-associated macrophages (yet not on cytotoxic T cells, natural killer cells and dendritic cells). And Xiongbin Lu, Xinna Zhang and colleagues used breast-tumour organoids co-cultured with tumour-antigen-specific cytotoxic $\mathrm{T}$ cells to identify epigenetic inhibitors that promote antigen presentation by class-I major histocompatibility complexes and that potentiate T-cell-mediated cytotoxicity.

All of these approaches to fighting tumour resistance or evasion are preclinical and provide, for the most part, proof-of-concept evidence of functionality and effectiveness. And many more are being conceived to make the tumour microenvironment more conducive to tumour suppression, to control the activation of effector $\mathrm{T}$ cells to limit off-tumour toxicity, or to increase their intratumoural accumulation or augment their persistence. And all of them will surely need to be adapted to resist or evade the many awaiting translational hurdles.

Published online: 12 November 2021 https://doi.org/10.1038/s41551-021-00825-7 